\title{
Funcionamiento psíquico de los niños con alopecia areata y vivencia de la enfermedad
}

\section{Children coping with alopecia areata, and their psychological functioning}

\author{
Alba Auxilio Vanegas Arbeláez*
}

\section{Resumen}

En este artículo se presentan los resultados de una investigación cualitativa que tuvo como objetivos: comprender el funcionamiento psíquico de los niños con alopecia areata y la vivencia de la enfermedad en los niños y sus familias. La muestra se conformó con seis niños diagnosticados con alopecia areata, y sus padres, todos ellos integrantes de una Fundación de la ciudad de Medellín que agrupa personas con este tipo de enfermedad; para la recolección de información se usó la entrevista semiestructurada y la prueba proyectiva Machover con los niños. El análisis de la información permitió concluir que existe un funcionamiento propio en los niños con esta enfermedad, caracterizado por la primacía del otro y del superyó, razón por la cual en las relaciones con los demás se asume una posición pasiva y se ajusta el comportamiento a las demandas superyóicas, se establece una inhibición para hablar de los sentimientos propios, un control rígido de los impulsos, dentro de ellos la agresividad, y se erigen ideales respecto al desempeño propio que al no ser satisfechos introducen la inconformidad consigo mismo. En la vivencia de la enfermedad por parte de los niños, se observa la interrelación entre la dimensión subjetiva y social de la enfermedad, puesto que las particularidades de su funcionamiento psíquico inciden en los obstáculos que tiene el niño para afrontar la reacción de los demás ante su alopecia areata. Por último, para los padres la

\footnotetext{
* Psicóloga U de A, candidata a Magister en Psicología, docente Uniminuto. Correo: vanegas.arbelaez@gmail.com
} 
vivencia de la enfermedad demuestra ser una tarea con varios frentes: debe afrontar lo que la enfermedad genera en sí mismo, debe contender lo que la enfermedad genera en los demás, y debe ser el bastión para que su hijo encare los efectos de su propia afección.

Palabras clave: psicosomática infantil, alopecia areata, funcionamiento psíquico, vivencia de la enfermedad.

\section{Abstract}

This paper presents qualitative research findings that aimed at understanding the psychological functioning of children with Alopecia Areata and the experience of this disease in children and their families. Data consisted of 6 children diagnosed with alopecia areata and their parents, all of them members of a foundation of Medellin city, which supports people with this type of disease. Semi-structured interviews and Machover projective test were used to take the data. It was concluded, there is an own operation in children with the disease, characterized by the primacy of the id and the superego, reason why they assume a passive position in relationships, and their behavior is conformed to the superego demands. Inhibition is established to express own feelings, a rigid control of impulses, aggressiveness among them, and ideal representations in relation to the own performance are raised, and if these representations are not satisfied, it may lead to generate a non-conformity with themselves.

Children coping with alopesia aleata, shows the interrelationship between the subjective and social dimension of the disease, since the peculiarities of its psychic functioning increase the difficulties to face the others reaction before the illness. Finally, it is evidence that dealing with the disease is a hard task for parents, they must face what the disease generates itself, what it generates in the others, and they should be the stronghold for their child to deal with his or her own condition. 
Keywords: childhood psychosomatic, alopecia areata, psychological functioning, experience of the disease

\section{Introducción}

Las afecciones psicosomáticas infantiles se encuentran asociadas "a una relación madre-hijo distorsionada" (Békei, 1992, p. 547). Kreisler (1985) también considera que la madre tiene una responsabilidad en la estructuración de la enfermedad, básicamente por su forma de asumir el ejercicio de la función materna, que en estos casos se caracterizaría por la vacuidad afectiva. La revisión de antecedentes demostró cómo estos postulados teóricos habían influenciado el desarrollo de investigaciones, que enfatizan en el análisis de las vicisitudes de la díada madre e hijo relativas a la enfermedad (Builes \& Carmona, 1993; Jurado \& Ramírez, 1997; Pasteur, Cuynet \& Mariage, 2010; Sierra \& Torres, 1987; Thomé, Campio \& Zogbi, 2006). No obstante, y sin desconocer los aportes de estos estudios, se considera que al centrarse en la relación madre e hijo no se profundiza lo suficiente en aspectos subjetivos del niño, y aunque algunas investigaciones intentan hacerlo, verbigracia el estudio de Schmitt, et al. (2011), en lo que enfatizan es en la enfermedad psicosomática, como factor de riesgo para el desarrollo de problemas mentales como la depresión, los trastornos de conducta y el déficit de atención con hiperactividad.

A razón de lo anterior, el estudio que se presenta en este artículo, puso énfasis en los aspectos subjetivos del niño con alopecia areata, enfermedad que de acuerdo con Farajzadeh et al. (2013), y Kakourou, Karachristou y Chrousos (2005) ha sido poco estudiada en la infancia y poco analizadas sus características clínicas, particularmente las psicológicas; así mismo, el estudio se orientó en la comprensión de la vivencia de la enfermedad en los niños y sus familias, puesto que uno de los pocos estudios encontrados sobre este particular 
concluyó que la alopecia areata genera en los niños dificultades en la formación de la identidad y en el entorno escolar, así como angustia en sus familias (Hunt \& Mchale, 2005).

En ese sentido se plantearon como objetivos generales de la investigación la comprensión del funcionamiento psíquico de los niños con alopecia areata, desde el análisis de la representación que de sí mismo habían construido, la caracterización de su dinámica relacional y la exposición de las manifestaciones emocionales; mientras que para la comprensión de la vivencia de la enfermedad, tanto en los niños como en sus familias, se consideró el análisis de la vivencia subjetiva en los niños con alopecia areata, y el análisis de la vivencia de la enfermedad en sus familias.

Por su parte, los conceptos que soportan teóricamente la investigación son: alopecia areata, término con el cual se denomina la caída de cabello que se sucede a pesar de que el folículo piloso aún se encuentra con vida y cuya sintomatología responde a unas pequeñas áreas de despoblamiento capilar que se presentan de manera repentina en la zona de la cabeza, las cejas, las pestañas o la barba; en su evolución estas pequeñas áreas pueden hacerse mayores y extenderse por toda la cabeza, produciendo la alopecia total, o incluso comprender otras partes del cuerpo como el vello corporal, a lo cual se le denomina alopecia universal. Cualquiera de estas alteraciones alopécicas afectan independiente de la edad, el género o la etnia del paciente.

Dentro de los aspectos más controvertidos de la alopecia areata se encuentra su etiología, muchos autores refieren que la causa de esta no se conoce con exactitud (Ghanizadeh, 2008), o que su etiología sigue siendo oscura (Güleç, Tanriverdi, Dürü, Saray \& Akgali, 2004); a pesar de ello, la mayoría de investigaciones revisadas sobre el tema se 
encuentran de acuerdo en afirmar que es una enfermedad multicausal donde intervienen factores psiquiátricos, psicológicos, autoinmunes, ambientales y genéticos (Alfani et al, 2012; Ghanizadeh, 2008; Olguín García, Martin del Campo, Rodríguez Acar \& Pedrero Peralta, 2013; Pérez, 1999) los cuales hacen de ella una enfermedad psicosomática de la piel (DíazAtienza \& Gurpegui 2011).

Psicosomática, concepto que a ojos de diferentes autores presenta dificultades para ser determinado, porque no existe un consenso sobre sus rasgos principales; no obstante, la lectura de autores como Békei (1992); Pedreira, Palanca, Sardinero y Martin (2001); Kreisler (1977) y Norberto Bleichmar y Celia Lieberman de Bleichmar (2001), permiten precisar para estos fines investigativos que dicho concepto se refiere a la relación establecida entre fenómenos somáticos y factores psicológicos, donde los últimos están conformados por una “estructura mental que impide asimilar un trauma psíquico de otro modo que no sea el somático" (Kreisler et al., 1977, p.27), y por un pensamiento operatorio desprovisto de una impronta afectiva (Kreisler et al., 1977). Desde el punto de vista etiológico, las afecciones psicosomáticas se originan en las fallas del vínculo temprano entre la madre y su hijo, las cuales están asociadas a la carencia de medios maternos que obstaculizan la integración de las funciones psicológicas y somáticas del niño y/o a la satisfacción de sus necesidades físicas. Y desde el punto de vista clínico, se descarta el sentido primario que algunos teóricos le asignan a las manifestaciones psicosomáticas.

Respecto a la psicosomática infantil, lo primero a tenerse en cuenta es que las enfermedades deben ser estudiadas teniendo en cuenta el periodo evolutivo en el cual se hacen presentes, porque algunas manifestaciones son más frecuentes en determinadas épocas, por lo tanto su análisis debe ligarse a la etapa en la que se desarrolle. El análisis de los niños 
debe concebirse desde la idea de un funcionamiento psíquico particular, mas no de una estructura psíquica, dado que la organización psíquica desde el punto de vista estructural se consolida en la adolescencia. Finalmente, y retomando a De Ajuriaguerra (1977), se debe tener presente el estudio de las emociones y los afectos para la comprensión de la psicosomática infantil.

El funcionamiento mental se concibe como un modo del psiquismo, que por la época a la que se circunscribe, esto es la infancia, se refiere al nivel que el niño ha alcanzado en su organización psíquica, básicamente en la representación de sí mismo, lo vincular y lo emocional. En contraposición a ello, la noción de estructura psíquica, se asocia a la consolidación de una organización poco variable en el tiempo.

Para la representación de sí mismo debe tenerse en cuenta que esta se asocia a la forma como una persona se designa a sí misma, y estaría conformada por el autoconcepto; la autoestima; las capacidades, ideales y ambiciones, y el autocentramiento en las relaciones con los otros. El autoconcepto sería la idea consciente o inconsciente que se tiene de sí; la autoestima indica la valoración que se tiene de sí mismo, de las relaciones, actividades y expectativas propias; las ambiciones e ideales son la posibilidad de desear para sí determinadas situaciones, y las capacidades son la forma cómo se piensa el desempeño en la realización de actividades. El autocentramiento en las relaciones con los otros da cuenta de la manera como el otro se sitúa ante los demás.

La dimensión del vínculo en este caso alude tanto a las relaciones interpersonales como a la estructura intrapsíquica que corresponde a las relaciones objetales. Las primeras se equiparan a la interacción que se tiene con el otro en el día a día, las segundas a una estructura intrapsíquica fundamentada en la representación que se ha construido del otro y de sí mismo. 
A pesar de la diferencia, ambas categorías guardan relación, porque en las relaciones interpersonales se hacen visibles aspectos de la estructura intrapsíquica que corresponde a las relaciones objetales (Coderch, 1990).

La dimensión emocional, que de paso puede decirse es fundamental en las relaciones objetales, son los afectos de amor, odio, agresión, tristeza, ternura, culpa y nostalgia (Kernberg, 1999).

La vivencia de la enfermedad se apoyó en los postulados de Laín Entralgo (1960), quien expone que la enfermedad es una experiencia o una vivencia para el enfermo, porque constituye un conjunto de vivencias reales que afectan tanto al organismo, como a quien la padece. En la dimensión del padecimiento de su enfermedad el paciente despliega procesos sentimentales e interpretativos, los más comunes son la aflicción, la amenaza, la soledad y el recurso y, en los segundos, el castigo, el azar, el reto y la prueba.

La aflicción está en relación con el malestar que incomprensiblemente adviene en un momento de la vida. La amenaza surge porque aparece de forma más consistente el riesgo de morir. La soledad se presenta porque la enfermedad impone un trato diferente entre el enfermo y los otros, y porque solamente él siente las dolencias y los padecimientos. El recurso es la ganancia secundaria que de la enfermedad se desprende.

En torno al castigo, Laín Entralgo (1960) expone que responde a una concepción punitiva de la enfermedad. La interpretación de la enfermedad como efecto del azar, comporta la idea del destino del hombre que por naturaleza enferma, a la vez que representa la posibilidad individual de caer enfermo. En la afrontación de la enfermedad como reto, lo que 
se pone de presente es el interés de curar o corregir el mal que se ha establecido, para lo cual pueden usarse dos procedimientos: los técnicos o los mágicos. Finalmente, la representación de la enfermedad como prueba parte del supuesto de que el paciente merece dicha dolencia; por lo tanto, lo que se pone a prueba es la paciencia y la magnanimidad del enfermo.

De acuerdo con la lectura de Mercado Martínez (1996), en las teorizaciones hechas por Arthur Kleinman se parte de la idea de que la enfermedad y el padecimiento son conceptos explicativos que hacen parte de un modelo más amplio, el cual en el caso del enfermo sería un modelo para explicar su padecimiento. Este modelo responde al "conjunto de creencias o nociones empleadas por quienes están involucrados en los procesos clínicos, e incluye uno o más de los siguientes puntos: etiología, aparición de los síntomas, fisiopatología, curso del padecimiento y tratamiento" (p. 54); ahora bien, dicho modelo solo tiene sentido dentro de una realidad social determinada.

\section{Método}

El estudio se enmarca dentro de la investigación cualitativa, dado que esta permite realizar un acercamiento al "significado o naturaleza de la experiencia de personas con enfermedad crónica, adicción, divorcio (...) y obtener detalles complejos de algunos fenómenos, tales como sentimientos, procesos de pensamiento y emociones" (Straus y Corbin, 2002, p. 12), caso específico de la investigación que analiza la vivencia de la alopecia areata como enfermedad, y aspectos como afectos, sentimientos y las representaciones que tanto de sí mismo y de los demás habían construido los niños. Siguió los lineamientos del enfoque fenomenológico-hermeneútico que, de acuerdo con Martínez (1996), “se centra en el estudio de [aquellas] realidades vivenciales que son poco comunicables, pero que son determinantes para la comprensión de la vida psíquica de cada persona" (p. 169). En el caso concreto de la 
investigación, el fenómeno analizado fue el funcionamiento psíquico de los niños con alopecia areata, así como la vivencia de la enfermedad por parte de ellos y sus familias; por lo tanto, se tomó en cuenta la percepción de los niños afectados y de sus padres. Para el diseño se eligió el Estudio de caso colectivo, según Galeano (2007) esta estrategia se refiere a la recolección, análisis y presentación detallada y estructurada de información sobre un fenómeno, una población o una condición general, con el objetivo de proporcionar mayor conocimiento sobre un tema, a partir del análisis de varios casos que comparten una misma característica, pero que no enfatiza en la generalización de los resultados a contextos más amplios, ni en la recolección de información sobre grandes cantidades de participantes.

\section{Participantes}

Los participantes de la investigación fueron seis niños y sus respectivos padres, pertenecientes a una fundación de la ciudad de Medellín que agrupa personas con alopecia areata. Los niños tenían edades entre los 6 y los 12 años de edad, diagnóstico médico de la enfermedad y asintieron participar de la investigación, una vez que los padres habían dado el aval para su participación, así como su consentimiento para ser entrevistados en calidad de progenitores. A pesar de que inicialmente se pretendía trabajar con los niños y la pareja parental, esto solo fue posible en uno de los casos, en los cinco restantes se entrevistó únicamente al niño y su madre.

\section{Instrumentos}

Para el proceso de recolección de información se emplearon la entrevista semiestructura con padres y niños y el dibujo de la figura humana con los niños. En total se realizaron 23 
entrevistas, de las cuales doce fueron con los padres; es decir, dos con cada una de las parejas parentales y once con los niños; solo con uno de ellos no fue posible realizar las dos entrevistas que si se hicieron con el resto de los pequeños. En cuanto a la prueba proyectiva se aclara que su uso estuvo argumentado por la oportunidad que brinda de aproximarse a algunos elementos inconscientes que dan cuenta del concepto que de sí mismo ha construido el sujeto, de sus relaciones con los otros, del manejo de sus impulsos y emociones. En este caso el número total de pruebas aplicadas fueron seis.

En la aplicación de la prueba se siguió el protocolo propuesto por Machover (1947) que sugiere tres fases a saber: indicación de la realización de la prueba con la consigna “dibuja una persona”, solicitud de realizar otro dibujo de un género opuesto al creado inicialmente y, por último, invitación al evaluado para hablar de los dibujos construyendo una historia sobre ellos o, en su defecto, planteamiento de algunas de las preguntas que hacen parte del cuestionario aportado por la misma prueba.

\section{Procedimiento}

En la recolección de la información primero se realizaron las entrevistas con los padres y se iniciaron los encuentros con los niños con la prueba proyectiva para luego continuar con las entrevistas. Con esta forma de proceder se facilitó el acercamiento al niño y se evitó que la prueba proyectiva se contaminara con lo abordado en las entrevistas. Todo el proceso se desarrolló en un lugar privado, donde solo estaban los participantes con quienes se iba a trabajar en ese momento; es decir, los niños no se hicieron presentes durante las entrevistas con los padres, y viceversa. 
Las entrevistas y la aplicación de la prueba se registraron en audio, mientras que la observación del comportamiento del niño y de los aspectos formales de la prueba como el tiempo de ejecución, el género del dibujo realizado en primer lugar, la secuencia seguida al momento de graficar cada una de las partes del cuerpo, etc. se registraron manualmente.

La información registrada en audio se transcribió, el almacenamiento de estos textos se orientó por la procedencia de cada uno de ellos; así se crearon tres bases de datos: la primera contenía las entrevistas efectuadas con los padres, la segunda las entrevistadas realizadas con los niños y la tercera los dibujos con las historias o las respuestas dadas sobre las mismas representaciones. En todos los textos se emplearon códigos para proteger la identidad de los participantes.

En el análisis de las entrevistas se respetaron las bases de datos creadas en la fase de almacenamiento, lo que quiere decir que se examinó el conjunto de entrevistas de los padres, aparte del conjunto de entrevistas de los niños. En ambos casos se siguió el proceso sugerido por Strauss y Corbin (2002) sobre codificación abierta y codificación axial. En la primera fase se segmentaron los documentos en unidades de sentido y se le asignó a cada una de ellas un nombre que diera cuenta de las propiedades y dimensiones de los datos; posteriormente se identificaron los conceptos que daban cuenta de un fenómeno; es decir, las categorías, así como cada uno de los elementos que hacían parte de ella, o lo que es lo mismo, las subcategorías; en la segunda fase se buscaron las relaciones entre las categorías. De esta forma se aislaron las siguientes categorías: funcionamiento psíquico, manifestación de afectos, relaciones con los otros, representación de sí mismo, vivencia de la enfermedad en los niños, explicaciones causales, afectación personal, vivencia de la enfermedad en los padres, manifestaciones físicas y reacciones emocionales, teorías explicativas sobre la caída 
del cabello, búsqueda de intervención médica, actitudes de los padres frente a la alopecia de sus hijos. Este procedimiento se elaboró en su totalidad en el software de investigación cualitativa Atlas-ti.

Para el análisis de la prueba se tomaron en cuenta las mismas categorías y subcategorías sugeridas por Machover (1974): Aspectos formales y estructurales del dibujo, aspectos del contenido, rasgos sociales; el cuello, los rasgos del contacto, rasgos misceláneos del cuerpo; la ropa, objetos y naturaleza; indicadores de conflicto y el tratamiento diferencial dado a las figuras del hombre y la mujer, el análisis de estas categorías se complementó con los postulados de Koppitz (1976), sobre los elementos de los dibujos que deben estar presentes de acuerdo con la edad del niño evaluado y los rasgos de las representaciones que son considerados como indicadores clínicos.

Con estas categorías se elaboró un formato para el análisis de cada uno de los Machover realizado por los niños, en el que a su vez se agregó un apartado conclusivo que presentara los hallazgos principales respecto al área afectiva, social, intelectual y de indicadores clínicos. Una vez concluido el análisis individual, se procedió al análisis general y se creó una base de datos en Excel en la que se consignaron las características de cada una de las categorías evaluadas en el contexto personal, para luego establecer cuáles de ellas eran comunes en los doce dibujos.

Para las historias y/o las asociaciones construidas respecto a los dibujos, se acogió la propuesta de Portuondo (2012) y por esa razón se aisló el tema de la historia, los matices o manifestaciones afectivas de esta; los personajes y el medio ambiente; y los indicadores de una historia conflictiva como la demora en iniciarla, o la dificultad para inventarla. En este 
caso se siguió el mismo procedimiento usado con los Machover: primero se analizaron las historias en el plano individual y luego en una tabla de Excel se expusieron las cualidades más representativas de cada uno de los ítems valorados en el primer análisis.

En la interpretación de los datos se compararon los sistemas categoriales extraídos de cada una de las tres bases de datos, a saber: entrevistas con los padres, entrevistas con los niños y análisis de la prueba proyectiva. Este proceso se realizó en Excel y permitió la ubicación de las categorías que se encontraban en las tres unidades hermenéuticas, las que se desarrollaron en dos de ellas, o en una sola. La única fuente de datos que no se tuvo en cuenta para la interpretación fueron los análisis de las historias, o de las asociaciones construidas sobre los dibujos, porque sus resultados eran tan disímiles que no permitían la ubicación de cualidades generales.

El proceso de validación de los análisis se complementó con la socialización de los hallazgos llevada a cabo en las sesiones de la línea teórica de Clínica y Salud a la cual pertenecía la investigación. La participación en un curso virtual sobre psicosomática infantojuvenil desarrollado en otro país suramericano y el conocimiento de disertaciones clínicas sobre pacientes con trastornos cutáneos, también confirieron soporte a algunas conclusiones abstraídas de las relaciones de los datos.

Finalmente, todo el estudio se sustentó en la Ley 1090 del 2006, específicamente su Título VII, y la resolución 008430 de 1993 expedida por el Ministerio de Salud. De acuerdo con lo expuesto en ellos se tomaron las siguientes disposiciones: se protegió la privacidad de los participantes al usar códigos como reemplazo de los nombres propios de las personas, lugares e instituciones que eran mencionados a lo largo de las entrevistas. Se realizó el 
consentimiento y el asentimiento informado siguiendo los parámetros que la resolución establece para cada uno de estos casos. Se clasificó la investigación de acuerdo con el riesgo al que estarían expuestos los individuos y se le otorgó un nivel mínimo de riesgo a todo el estudio. Se firmó el consentimiento informado con cada uno de los padres, tanto en calidad de participantes de la investigación, y como representantes legales de los menores; con los niños se firmó el asentimiento informado.

\section{Resultados}

La presentación de los principales resultados seguirá el orden que ya se ha señalado respecto a los dos nodos de la investigación: funcionamiento psíquico de los niños con alopecia areata y vivencia de la enfermedad en los niños y sus familias.

\section{Funcionamiento psíquico de los niños con alopecia areata}

En la representación que de sí mismo han construido los niños se evidencia un adecuado reconocimiento del género propio y aceptación de la condición sexual y social de ser hombre o mujer; la prueba de ello es que en la realización de los dibujos todos los niños iniciaron con la representación de una figura que coincidía con el sexo del niño dibujante; la mayoría le dio un tratamiento diferencial a las figuras del varón y la mujer, incluyendo en ellas elementos que permitían reseñar el dibujo como representante de uno u otro género.

Por la consideración que se hace de las cualidades propias, se infiere una representación valorizada de sí mismo, lo cual incluye la idea de ser respetuoso, tener un buen desempeño intelectual y ser obediente. La percepción del buen desempeño académico se ve como una característica valorada por los otros y, de acuerdo con la visión de los niños, influye en el amor que los padres les manifiestan: "Ent.: ajá, entonces tú dices que la mami te quiere 
porque tú eres muy juicioso, y ¿por qué más la mamá te quiere? (...) / Porque soy muy bien estudiante" (Unidad hermenéutica Entrevistas Niños [U-H-E-N], P1, L. 025). Sin embargo, contrasta con las disconformidades que de manera proyectiva se manifestaron sobre el desempeño académico y que dan la idea de exigencias que se ejercen sobre sí mismo (ver aire grotesco de los rasgo faciales en la Figura 5, la Figura 11 y Figura 12, y las diferencias de tamaño de las cabezas en las Figuras 7 y 8, caso en el cual se le asignó mayor dominio intelectual a la figura del hombre, a pesar de que quien dibujada era mujer).

Por su parte, la representación de sí mismo como una persona obediente responde a la idea de alguien que se ajusta a disposiciones que el otro ha determinado, lo que se acompaña de un reconocimiento de las figuras de autoridad, del sentido de las normas y de una facilidad para el cumplimiento de ellas. Esta cualidad se consolida con las palabras de los padres al respecto, para muchos de ellos sus hijos son juiciosos y en general cumplen con las normas establecidas, aunque en algunas ocasiones se les debe recordar los deberes por realizar:

(...), pues a ella no hay necesidad de que uno la tenga que regañar mucho, ni como pegarle, no, pues ella no es grosera, ni nada, pero lo más bueno de ella es que no es contestona, ni nada, ella es muy querida pa' uno manejarla (Unidad hermenéutica Entrevistas Padres, [U-HE-P] P7, L. 027).

Respecto a la manifestación de afectos, esta se encuentra marcada por tres condiciones: poca expresividad, expresividad en el contexto familiar y sofocamiento de la agresividad. La poca expresividad en algunas ocasiones se nota desde épocas tempranas y se va consolidando en etapas posteriores, esta comprende la dificultad para demandarle al otro algo que se necesita, así como la incapacidad para hablar de lo que se siente: 
[Mi hija] me parece muy poco expresiva, demasiado diría yo, pero yo a veces veo que no es ni siquiera que no sienta algo, sino como que no es capaz de expresarlo fácilmente, o sea, yo a veces veo que ella es como con ganas, como con impulso, pero como que algo la para (U-H-EP, P10, L. 048).

Las palabras de los niños al respecto reportan además de su dificultad para expresarse, las explicaciones sobre el origen de esta actitud que ellos mismos catalogan como una incapacidad para hablar de lo que se siente: "pues yo no tengo la costumbre, pues yo no sé, no soy capaz, yo como que no me desahogo con nadie, entonces yo mis miedos que tengo adentro los dejo para mi solita" (U-H-E-N, P7, L. 368) Pero en otras ocasiones, la inexpresividad está articulada al deseo de que el otro no se entere de las afectaciones que se experimentan en el día a día, o al sofocamiento del malestar por razones que obedecen a un orden más moral y/o normativo.

"Ent.: le preguntan [a una persona] si está triste y estando triste dice que no, que no pasa nada, ¿por qué lo haría esa persona? / Porque no quiere como que nadie sepa, no sé” (U-H-E-N, P9, L. 050).

"Ent.: ¿qué haces con esa rabia M.? / Nada, me toca quedarme quieto, calladito / ¿Y eso? / Ah, porque después pueden pasar cosas peores" (U-H-E-N, P4, L. 078).

Lo expresado hasta el momento encuentra su correlato en las producciones gráficas del test; por ello, la conclusión general sobre el área afectiva establece que en la mayoría de los casos se presenta una tendencia a inhibir la expresión de emociones y sentimientos. El sustento de este colofón son los rasgos estampados en los dibujos a las zonas responsables de la comunicación que básicamente son: asimetría en la curva de la boca (ver Figura 5); tamaño 
pequeño de los dibujos (ver Figura 3 y Figura 4), y la ubicación de las dibujos en la zona superior, lado derecho de la hoja, particularidades últimas que se presentaron en la mayoría de ellos (ver Figura 3, Figura 4, Figura 5, Figura 6 y Figura 9).

A pesar de lo presentado, el análisis de los resultados también anotició de la expresión de emociones y sentimientos pero solo en el contexto familiar, lo cual no significa una ambigüedad en la lectura de los datos, sino que indica las condiciones bajo las cuales el niño da a saber lo que siente. Los padres sugieren momentos en que los niños les comunican sus diversos estados de ánimo bien sea por el uso de palabras o de gestos que ellos deben interpretar: "él es muy serio, o sea, él como que siempre está riéndose no, pero si se le ve la carita, pues ¿cómo qué te puedo decir yo? Sí baja como la intensidad de sus acciones” (U-HE-P, P2, L. 216).

Por las particularidades descritas se decidió nombrar esta categoría como Expresividad en el ámbito familiar, porque con ella se marca una diferencia entre este ámbito y el social, en este último se notan claramente algunas dificultades, que no sólo se establecen por las características de la manifestación de afectos de los niños entrevistados, sino también por la relación que se construye con los otros.

La última característica de la manifestación de afectos es el sofocamiento de la agresividad. El análisis del test sugiere la presencia de tendencias agresivas por las diversas cualidades que se observan en los doce dibujos analizados. En algunos de ellos, lo que sobresale es la forma gruesa de la línea (ver Figura 7, Figura 8, Figura 9, Figura 10, Figura 11 y Figura 12); en otros, la representación de los pies descalzos (ver Figura 5), o el gran tamaño de las figuras (ver Figura 7 y Figura 8); en la Figura 1 y la Figura 2 la presencia de línea de 
apoyo dentada, en la Figura 6 y en la Figura 9 la omisión de la nariz, y en la Figura 12 la representación de abundante lluvia. No obstante, lo que las palabras de los padres precisan es que el tipo de conducta agresiva se limita a situaciones familiares en las que el niño manifiesta por medio de gestos su rabia con los demás:

Primero tenía el vicio como [de] empuñar la mano, y como ¡ay!, como de impotencia, (...) entonces uno la sentía como jay!, como de la rabia, como no puedo hacer nada, o le voltea a uno los ojos. Mi mamá le decía algo y ahí mismo le voltea los ojos, y mi mamá con eso tenía pa' llamarme: (...) esa muchacha tan grosera, uno se voltea y lo mira a uno feo porque uno la regaña (U-H-E-P, P10, L. 127).

Esta exteriorización de las tendencias agresivas cumplen la función de dar a saber un malestar, pero también da cuenta del alejamiento de hechos violentos que puedan dañar al otro. En las descripciones de los padres sobre las relaciones sociales de sus hijos, la agresividad consumada en hechos violentos no hace parte de las actitudes del niño hacía los demás, lo que se resalta es la existencia de encuentros interpersonales tranquilos y afectuosos tanto con los pares como con las personas adultas.

Ella no es de peleas, nunca lo ha sido, ni siquiera con el hermano, él le pega y ella se queda quieta, ella no es de pelea, ella no es de problemas, ella (...) es amor y paz, ella es tranquila, es eso, armonía (U-H-E-P, P4, L. 336).

Con lo desarrollado surge la pregunta sobre la existencia de una vivencia más interna de la agresividad. Los relatos de los niños dejan entrever una vivencia en solitario de las 
situaciones que producen en ellos rabia y el advenimiento de sentimientos de malestar con ellos mismos por situaciones en las cuales han contrariado al otro:

Ent.: ¿Qué le pasará a una persona, de acuerdo a tu experiencia, cuando se guarda esa rabia? / Pues a veces como que me pongo a pensar: ¡ay! ¿yo por qué grité eso? ¿Yo por qué dije eso? Como un rencor, no sé, así / ¿Y rencor con quién? / Contra mí misma (U-H-E-N, P7, L. 434).

Lo presentado muestra más la influencia de la función superyoica en el control de los afectos, que la agresión puesta sobre sí mismo. Esta afirmación, puesta en perspectiva con los demás casos, se hace más consistente al encontrar rasgos en los dibujos del test que refieren la presencia de un control rígido de los impulsos (ver forma del cuello en las Figuras 1, 2, 4, 6 y 11). Así, la hipótesis que se consolida es que las tendencias agresivas se trasmudan por efecto del superyó en ausencia de agresividad manifiesta, con lo cual se silencian dichas tendencias, pero no se las extingue.

Respecto a lo vincular, que congrega las relaciones familiares y sociales, puede decirse que la familia es considerada como lo más importante de la vida y las relaciones sociales como un vínculo que representa dificultades para el niño. En los aspectos familiares sobresale la relación establecida por los niños con sus padres, en general, tanto padre y madre son vistos como personas amorosas que buscan el bienestar de sus hijos, lo que a su vez se desprende de las expresiones de cariño, cuidado y complacencia que los niños reciben de ellos. Esta situación favorece el establecimiento de relaciones de dependencia que en ocasiones empaña la posibilidad de decidir por sí mismo y de concebir una vida sin sus progenitores. Justamente, frente a este último aspecto surge la angustia por la pérdida imaginada de figuras significativas, con lo cual se hace alusión a los estados de preocupación que advienen 
en los niños por la posibilidad de perder a uno de sus seres allegados. Las palabras de algunos de ellos al respecto son: "Ent.: por qué te da rabia que el papá no esté? / De pronto muere” (UH-E-N, P11, L. 078).

En las relaciones con los demás miembros de la familia extensa, resalta la riqueza de los vínculos establecidos con cada uno de ellos. La mención al contacto con los abuelos se circunscribe a la diversión y el cuidado que ellos ofrecen; los tíos son vistos como aquellos que además de dar un buen trato, también están dispuestos al juego con sus sobrinos; los primos son sinónimo de juego, y los hermanos de buen trato, pero también de altercados generados por las bromas que entre ellos se hacen.

En las relaciones sociales con personas externas a la familia aparecen nuevamente las diferencias que se señalaban en la exteriorización de sentimientos y emociones en el ámbito familiar y social. La mayoría de los padres se encuentran de acuerdo al afirmar que sus hijos son tímidos, por esta razón sus relaciones sociales están marcadas por la inhibición: “obviamente llega a un lugar y es como tímida, como todo niño, o en algunos casos, porque hay unos que no realmente, entonces ella mira analiza y todo eso, (...) es timidez se le ve" (U-H-E-P, P4, L. 241). La timidez nombrada por los padres se revela en los dibujos sobre la figura humana, con la forma cómo se representaron la boca, los brazos y las manos. La boca por lo general se graficó con una línea ancha vuelta hacia arriba (ver Figura 3, Figura 4, Figura 7, Figura 8, Figura 11 y Figura 12). Los brazos y las manos cuentan con diferentes rasgos que aluden a las dificultades en el contacto con los otros: la ausencia de las manos (ver Figura 11 y Figura 12); la poca demarcación de las mismas (ver Figura 9 y Figura 10); la asimetría en los brazos (ver Figura 7); y la tendencia a ubicar los brazos cerca al tronco, como 
colgando flácidamente al lado de la figura (ver Figura 3, Figura 5, Figura 6, Figura 9, Figura $10)$.

Ahora bien, las dificultades existentes no riñen con la posibilidad de que el niño entable relaciones cordiales y respetuosas con los demás, que también denotan una actitud pasiva y sumisa con el otro. Este aspecto, aunado a lo visto sobre el sofocamiento de la agresividad, y lo avizorado frente al movimiento en el cual oscilan los niños en sus relaciones sociales: dificultades en el contacto y trato respetuoso y cordial, da cuenta del intento del niño por ajustar sus obstáculos a lo exigido socialmente, lo que finalmente es otro triunfo del superyó.

\section{Vivencia de la enfermedad}

En la vivencia de la enfermedad se tomaron en cuenta tanto las experiencias de los padres respecto a la alopecia de sus hijos, y las vivencias de los niños derivadas de su enfermedad.

Las experiencias de los padres frente a la enfermedad de sus hijos alude a un proceso que inicia con la pérdida repentina de cabello en los niños y las reacciones emocionales que se suceden en los progenitores a raíz de dicha pérdida; el proceso se continúa con la construcción de teorías explicativas para dar cuenta de las causas de la caída del pelo y con la búsqueda de intervención médica. Todas las etapas mencionadas se acompañan con una diversidad de actitudes que van desde la dificultad para afrontar los acontecimientos, a una aparente superación de los mismos. En este primer encuentro con la enfermedad, la mayoría de los niños quedaron excluidos de la situación como sujetos activos, porque sus cortas edades al momento de aparición de la alopecia (entre 9 meses y 2 años de edad) no les permitían darse 
cuenta de los cambios que se empezaron a evidenciar en su piel. Por ello, son sus padres los que vivencian los estados de tensión emocional producto de tales alteraciones.

El avistamiento de las pequeñas zonas despobladas de cabello en la cabeza de sus hijos se acompaña de pena, rabia, miedo, tristeza, llanto y angustia. La pena y la rabia se originan por los interrogantes frente al encuentro con los otros:

Inicialmente fue pena, no lo voy a negar, a mí me daba pena, me daba miedo, me daba temor, yo no sabía cómo enfrentarlo, porque más que sentirme atacada era cómo afrontarlo para que la niña no se fuera a sentir ofendida, porque obviamente yo reaccionaba era con rabia, yo me ofuscaba y me enojaba (U-H-E-P, P6, L. 312).

La tristeza y el llanto surgen por el impacto personal y familiar, y la angustia por la incertidumbre frente a las causas de la pérdida repentina de cabello y de la gravedad de la alteración. Ante estas incertidumbres surgen las teorías explicativas que de acuerdo con el factor etiológico aislado pueden dividirse en los siguientes grupos: las que refieren procesos de orden biológico, las que consideran aspectos mágicos, y las que recurren a elementos psicológicos. Las primeras consideran infecciones y efectos de medicamentos como las causas de la alopecia; las segundas enfatizan en la mala mano del peluquero; y las terceras asocian la caída del cabello con el estrés y los miedos e inseguridades de la madre durante el embarazo y el postparto. Estas últimas teorías ya se encuentran permeadas por el discurso médico que ha dado a saber la relación entre alopecia areata y estrés, y finalmente es la teoría que gana terreno, incluso muchos padres la complementan asociándole aspectos personales del niño que de acuerdo con su visión van a favorecer la presencia del estrés, o realizan lecturas a posteriori de los hechos que rodearon la aparición de la enfermedad: 
Yo pienso que puede ser más fácil como por lo que ella no expresa, entonces como que se queda como con todo eso reprimido sea bueno o sea malo, como que ni dice nada bueno, ni dice nada malo (...), entonces de pronto puede ser estrés de la misma forma de ser de ella, que no es capaz como de sacar lo que siente (U-H-E-P, P10, L. 646).

Las teorías construidas también se acompañan de la búsqueda de intervención médica que siempre acerca a los padres al diagnóstico de la enfermedad y al inicio de tratamientos dermatológicos que posteriormente van a ser abandonados. Con el diagnóstico, los padres conocen el nombre de la enfermedad, la evolución de esta y acallan su intranquilidad al darse cuenta de que es una alteración que no reviste gravedad desde el punto de vista orgánico. El diagnóstico que trae calma para algunos padres, en otros genera extrañeza: “(...) que le digan a uno un nombre bien raro: que alopecia, pero ¿qué es eso? ¿Cómo que alopecia?” (U-H-E-P, P3, L. 362).

Frente a los tratamientos, los padres siempre acogen las recomendaciones dermatológicas, pero los efectos secundarios de estos tratamientos que algunos padres califican de torturantes, son la razón para abandonarlos;

(...) El doctor A. nos dijo: no pues, sirve, pero [con] el tratamiento [el pelo] le crece, pero vuelve y se le cae. Entonces ya, pues yo hablé con [mí esposa] no, no, pues es bobada seguirlo sometiendo a esa tortura, es más bien ya dejar de insistir con eso (U-H-E-P, P1, L. 646).

No obstante, la ilusión que se genera de que el niño recupere su cabello, es la causa para que los tratamientos se continúen, por esa razón se reemplaza la intervención médica con procedimientos naturales que traen más comodidad para los padres. 
Todo este proceso de afrontamiento de la enfermedad se acompaña de las actitudes de los padres frente a la alopecia de sus hijos, las cuales van desde la dificultad para afrontarla, hasta la aparente tranquilidad y superación de la misma. En las dificultades intervienen factores de índole personal y social. Los primeros se relacionan con las afectaciones producto de las modificaciones en la apariencia física lo que introduce la necesidad de renunciar a cualidades físicas que admiraban en sus hijos y al cumplimiento de fantasías construidas durante el embarazo, como adornar el cabello de su hija, así como asumir que su hija mujer no posee un cabello largo que la diferencie físicamente de los hombres:

\footnotetext{
Ah, pa' siempre, uno la ve más niña. Que muchos le dicen a uno: ¡ay tan muchachito!, tal cosa, primero le decían mucho niño, como que no le veían las areticas, o en fin, y yo: no, ella no es un niño (U-H-E-P, P8, L. 142).
}

A estas situaciones se le suman las preguntas de los otros que incomodan a los padres y a los niños: “y la gente pues preguntando ¿qué es eso? ¿Qué tiene? Sí, maluco”. (U-H-E-P, P12, L. 121). La mirada que irrespeta la privacidad del niño y, por último, las bromas y burlas de las que es objeto sobre todo en el ámbito escolar. Todas estas situaciones intervienen en la percepción de la alopecia como una condición que diferencia, lo que indudablemente afecta el proceso de aceptación de la enfermedad de sus hijos: "no es lo mismo, no es lo mismo uno ir con, con su niño común y corriente en el sentido de que tiene todo, no es lo mismo, a que ir con alguien que ya lleva algo diferente (U-H-E-P, P6, L. 452).

El reverso que se va dando en cada una de estas situaciones, es lo que permite mayor tranquilidad frente a la alopecia, incluso la idea de que la misma ha sido superada, aunque 
también algunos factores personales favorecen el cambio, por ejemplo, la posibilidad de ver lo positivo de los problemas, "hay muchas cosas positivas por el hecho de no tener cabello, no mires las negativas, hay muchas positivas" (U-H-E- P, P2, L. 960); así como el hecho de darle una valoración diferente al cabello y la forma como el niño sortea las dificultades que se presentan en su día a día. Ahora bien, aunque tienen un efecto de cambio en el afrontamiento de la enfermedad, estos factores no se traducen en la adquisición de un estado de total tranquilidad, al respecto se escuchan las siguientes palabras: “y ya prepararlo psicológicamente, para que tome la alopecia como con, no con digamos con naturalidad porque es muy complicado, pero que sí que la sepa llevar (U-H-E-P, P1, L. 653).

La conclusión es que los padres con esta forma de asimilar la enfermedad cumplen con una doble función: encontrar un punto intermedio entre las dificultades para aceptar la alopecia y la superación de la misma, y servir de semblante para ayudar al niño en su proceso de afrontamiento de su propia condición, situación que se evidencia en el apoyo brindado al niño que tiene la connotación de dar seguridad y ser sostén anímico y emocional.

De otro lado, las experiencias de los niños frente a su enfermedad están rodeadas de las dificultades que causa hablar de este evento, pero, a pesar de ello, en el proceso de afrontamiento pueden construir teorías que explican el origen de su condición. Para la mayoría de ellos las causas son externas: dios, la acción de un maleficio o el azar de la vida, “es que uno sabe qué le va a dar, porque mi tía no sabía pues como de que iba a sufrir [su hija] y ella sufre de asfixia, uno no sabe cómo ahí, que uno qué va a sufrir" (U-H-E-N, P9, L. $358)$. 
Además de estas teorías se encuentran las afectaciones que en este caso están directamente relacionadas al encuentro con los otros, que vía las burlas, los insultos y las bromas remarcan el rasgo que el niño porta. De esta situación se desprende la idea de ser diferente y con ello se menoscaba la seguridad en sí mismo:

Ent.: ¿qué sería lo más difícil para un niño que tenga alopecia areata? / ¿Difícil, algo difícil? Como hay niños, como acomplejados, como que les da miedo, pena o miedo salir y así. / Ent.: M. y ¿por qué les da miedo salir? / Como porque les van a decir algo. / Ent.: ¿qué les pueden decir? / Que, pues, así como hay veces me decían a mí, que siempre me decían: ay, usted tiene cáncer, o como discriminarlo (U-H-E-N, P9, L. 389).

Como era de esperarse, estas afectaciones se ven reflejadas en el plano corporal. En el análisis de los dibujos se observa cómo el cabello y las cejas son elementos del cuerpo donde reposan algunos conflictos, así lo indica: la alteración de la sucesión en la que se espera deben ser dibujadas las partes del cuerpo para dar énfasis al cabello; las borraduras en la zona del pelo y/o el énfasis que recibe, lo que claramente alude a ansiedades respecto a esta zona (ver Figura 3, Figura 4, Figura 7 y Figura 9).

Finalmente, algunos padres consideran que a pesar de estas situaciones los niños llevan un proceso que les permite asumir con más tranquilidad su condición; por ello, algunos toman la decisión de no ocultar su diferencia, mientras que otros se aferran al deseo de recuperar nuevamente su cabellera; debido a esto se concluye que: la vivencia de la enfermedad se encuentra ente la aceptación y la negación de la misma. 


\section{Discusión}

Los hallazgos de la investigación frente al funcionamiento psíquico conducen a defender la relación entre unas características psíquicas precisas y la presencia de la alopecia areata, razón por la cual el trabajo se distancia de aquellos estudios que le adjudican a las condiciones ambientales la causa etiológica de la enfermedad, verbigracia el trabajo de Díaz-Atienza y Gurpegui (2011), dichas características son: dificultad para la expresión de afectos; ausencia de agresividad manifiesta que contrasta con las tendencias agresivas inconscientes y ajuste del comportamiento a exigencias superyoicas; rasgos que se encuentran atravesados por el mismo hilo conductor: establecimiento de una relación pasiva con el otro.

Las dificultades para expresar los afectos son uno de las rasgos más comunes en los pacientes psicosomáticos, esta es la razón por la cual se han acuñado conceptos como el de alexitimia (Sifneos, 1972, como se citó en Sánchez, 2009) el cual alude a la existencia de un “(...) estilo cognitivo caracterizado por inhabilidad para verbalizar sentimientos y discriminarlos" (p. 52). El encuentro con los niños entrevistados permite poner en cuestión para su caso particular este estilo cognitivo, porque a pesar de que existe en ellos una inhibición para expresar lo que sienten, la referencia a las diferentes situaciones en las que han experimentado miedo, tristeza, ansiedad o alegría, dan noticia de su capacidad para diferenciar diversos tipos de sentimientos. Por esta razón, lo observado en los niños responde más a una dificultad en la expresión de afectos, que a la comprensión de estos, lo que técnicamente se denomina alexitimia de tipo expresivo. Esta característica, a la par de definir la manifestación de lo afectivo, también da cuenta de la relación que estos niños establecen con los otros, puesto que los impases en la traslación de los sentimientos a las palabras se traduce en un vínculo en el que la narración de las emociones propias no se realiza, en el que las experiencias afectivas y los diferentes estados emocionales que se atraviesan en el día a 
día, no se implican verbalmente en la relación; en consecuencia, el niño queda a la espera de que sea el otro quien indague por las marcas que algunas vivencias hayan dejado en el plano afectivo.

En medio de este panorama de afectos poco expresados, surge con fuerza la alusión a impulsos agresivos, que se han calificado de tendencias en estado latente porque a pesar de que existen evidencias de ellos, estos no se concretan en actos violentos que tengan el objetivo de destruir al otro. Winnicott (1964) al realizar una comparación entre los niños que se vuelven agresivos con facilidad, con aquellos que retienen la agresión, expresa que los primeros logran el alivio producto de la manifestación de la agresión y la hostilidad; mientras que los segundos no logran percatarse de que esta clase de sentimientos son limitados y gastables; además, la limitación en la expresión de afectos agresivos configura en el niño una actitud tensa, excesivamente contralada, que repercute en la inhibición de todos los impulsos y, por ende de la creatividad, aspecto que dentro de la teorización de este autor es de vital importancia porque se refiere a lo modos disponibles para enfrentar el principio de realidad (Winnicott, 1970).

De estas elucidaciones se extraen las siguientes conclusiones: el control excesivo sobre los impulsos solo impide que estos se manifiesten, pero no que puedan ser aliviados; esta situación si bien da cuenta de la capacidad del niño para pensar en el otro y protegerlo de actos que puedan dañarlo, la imposibilidad para hacer una descarga del impulso y transformarlo en algo efímero, lo deja a expensas de una agresividad que no tiene límite. Por otra parte, el apocamiento de la creatividad incidirá en la formación de una relación de sumisión con los otros, a razón de las limitantes para hacerle frente a las exigencias de la realidad exterior y de conservar un sentimiento de omnipotencia sobre ella. 
Así, emerge el carácter paradójico de la inhibición de la agresividad, dado que si bien beneficia al otro porque se le otorga una conducta exenta de irrupciones agresivas que puedan afectar la relación como tal y la representación que el niño guarda de ella, se pierde capacidad para dar resolución al principio de realidad, en la medida en que la posibilidad de construir formas para enfrentarlo se encuentra inhibida.

El mantenimiento de la agresividad en estado latente a la par de dar cuenta de la primacía que el otro tiene en el psiquismo del niño, también anoticia del influjo de la función superyóica en su vida anímica. Estos dos elementos del psiquismo son complementarios, toda vez que esta instancia se construye a partir del papel ejercido por un agente externo como la autoridad parental (Freud, 1933/2004b). La relación establecida permite concluir que para el caso particular de los niños entrevistados, el control ejercido sobre los impulsos agresivos hacen parte de un modo de funcionamiento psíquico más generalizable: el ajuste del comportamiento a las exigencias del superyó, que también se hace visible en el reconocimiento de los niños a las figuras de autoridad, en el respeto por el sentido de las normas, en la facilidad para el cumplimiento de las mismas y en las cualidades que valoran de sí, siendo una de estas la obediencia.

El superyó como instancia psíquica tiene como funciones la observación de sí, la conciencia moral y la función de ideal, y, dado hacer parte de un aparato psíquico construido por el yo y el ello, está en relación con el yo, al que observa y controla con normas de conducta (Freud, 1923/2004a). Si bien es claro que la construcción de esta instancia es una conquista que todo niño debe realizar, en los niños participantes de la investigación lo que se observa es el levantamiento de un superyó exigente que controla los impulsos, y que incluso 
obliga a cumplir ideales de perfección que no podrán ser saciados, trayendo como consecuencia inconformidades consigo mismo; ejemplo de esto es el contraste entre la realidad de un buen desempeño académico demostrada por la mayoría de los niños y la insatisfacción personal frente a este aspecto. De esta forma se erige un costo psíquico que en este caso es el yo quien lo paga. En los niños el costo se abona con la inhibición en la relación con el mundo exterior y con el alzamiento de ideales que desvalorizan los logros académicos obtenidos hasta el momento.

Frente a la vivencia de la enfermedad, se encontró que las conclusiones que establece este estudio son similares a las que se derivan del trabajo de Hunt y Mchale (2005), las más sobresalientes son las siguientes: los efectos negativos de la alopecia areata en el entorno escolar de los niños, la importancia de abordar la vivencia de la enfermedad desde el punto de vista familiar, y los cambios que se dan en las estrategias de afrontamiento de la enfermedad.

La experiencia de la enfermedad de los niños participantes de la investigación se encuentra atravesada por las particularidades de su funcionamiento psíquico; por tal razón, hablar de ella no es una tarea que se consuma con facilidad en una conversación, pero a pesar de ello, logran dar cuenta de la construcción realizada en torno a su manera de concebir y explicar la enfermedad.

Para la mayoría de los niños, el encuentro con sus pares significa afrontar la burla por tener su cabeza desprovista de cabello; en ese sentido, la forma cómo la enfermedad es percibida por los demás es uno de los principales obstáculos que los pequeños deben afrontar, porque desde su composición orgánica la alopecia areata no produce dolor, ni incapacidades que restrinjan el desempeño del niño en sus actividades diarias. Ahora bien, esta afirmación 
debe analizarse en relación con las dificultades que ya se mencionaron respecto al vínculo de los niños con los otros, porque este rasgo incide en la forma cómo se afronta la reacción de los demás ante una marca que diferencia. Por ello, los efectos de la enfermedad no pueden valorarse únicamente desde sus aspectos sociales, sino desde el interjuego que se establece entre la dimensión orgánica, subjetiva y social de la enfermedad.

En vista de lo anterior, se considera que la caída del cabello desemboca en concepciones personales que afectan la seguridad en sí mismo, y en rechazos inconscientes de los rasgos que la enfermedad ha marcado en el cuerpo; al mismo tiempo, las percepciones de los demás frente a la afección inciden en las concepciones que se construyen de sí; es decir, el efecto de este interjuego es el núcleo de la vivencia de la enfermedad por parte del niño, pero este a su vez está asociado a la dinámica de su funcionamiento psíquico.

La vivencia de la enfermedad en los padres demuestra que este es un proceso que también les afecta; la comprensión de los factores identificados en dicha vivencia permitió concluir que este proceso guarda relación tanto con los postulados sobre experiencia de la enfermedad propuestos por Kleinman (como se citó en Mercado, 1996) y por Lain (1960), como también con algunas concepciones del duelo propuestas por Worden (1997). En ambos casos, lo que se pone de presente es el intento de afrontar una situación que, debido a la pérdida que implica, genera incertidumbre, pero dado el efecto de las acciones emprendidas brinda la sensación de ser un evento superado.

Lain (1960) permite entender cómo los sentimientos inmediatos de amenaza y aflicción, que surgen en los momentos iniciales de la enfermedad, van dando paso a interpretaciones menos fatales de esta, primero porque la intervención médica trae como 
efecto una explicación sobre los orígenes de la enfermedad, y segundo porque el inicio del tratamiento erige la ilusión de una situación controlada que tendrá un desenlace favorable. A pesar de estas modificaciones, la experiencia de la enfermedad sigue acompañada de malestares por los efectos que el contexto social provoca la presencia de un niño sin pelo. Por esta razón, se afirma que la experiencia de la alopecia areata en los padres está permeada por la dimensión subjetiva y social de la enfermedad. De la subjetiva hacen parte las reacciones ante los primeros síntomas de la alteración física y las interpretaciones con las que se intenta comprender la razón de ser de esta; de la social, la forma cómo se enfrentan las respuestas de los otros ante la alteración física (Kleinman, como se citó en Mercado, 1996).

Así, asumir la alopecia areata de sus hijos implica para los padres una tarea de varias caras. Como padre debe afrontar los efectos que en sí mismo se derivan por los cambios físicos que se han suscitado en su hijo, debe contender lo que la enfermedad genera en el ámbito social, y, por último, debe ser el bastión para que su hijo establezca la forma de afrontar las dificultades producto de su enfermedad. Como efecto de estas labores, surge la ilusión de que la alopecia areata del niño es para los padres un evento superado; pero lo que se evidencia en la mayoría de los casos es que esta ilusión responde a la construcción de un semblante; algunos padres manifiestan al respecto que delante de su hijo la enfermedad es un evento superado.

La idea de un semblante remite en este caso al hecho de que los padres deben mostrar ante su hijo que las consecuencias de la enfermedad no generan dificultades. Este deber ser está sustentado en el papel que los padres tienen en la estructuración psíquica del niño. Winnicott (1980) considera que la madre ejerce la función de sostén emocional, que si bien es fundamental en los primeros meses de vida, debe prolongarse durante la infancia. Así el 
semblante cumple una función, crear en el niño la idea de que la situación puede afrontarse; sin embargo, también implica que en el trasfondo la actitud mostrada por los padres es diferente; es decir, la enfermedad con sus consecuencias sí genera dificultades en ellos. En ese sentido, el semblante sirve al niño, pero para el caso de los padres lo que se demuestra es que estos deben continuar con la resignificación de las pérdidas, por ejemplo, del ideal de un hijo sano y de un elemento que ya no permitirá el cumplimiento de fantasías construidas en la etapa de gestación, como adornar con peinados la cabeza de su hija, o adorar los rasgos físicos de su hijo, porque como lo enuncia Palacios "la enfermedad del niño afecta a la madre en su plano narcisista" (2013). En otras palabras, los padres deben vivir el duelo que la enfermedad implica.

\section{Conclusión}

Existe un funcionamiento psíquico propio de los niños con alopecia areata que se encuentra circunscrito a una representación de sí mismo que oscila entre la valoración de la obediencia y el respeto por la norma, y la inconformidad por el desempeño académico; a una dinámica relacional que denota una posición de sumisión al otro, y a una manifestación de afectos marcada por las dificultades para traducir en palabras sus sentimientos y emociones, y por la inhibición de sus tendencias agresivas. En ese sentido, la vida anímica de estos niños se encuentra dominada por la primacía del otro y del superyó: por esa razón, en la relación con los demás se asume una posición pasiva y se ajusta el comportamiento a las demandas superyóicas, lo que trae como consecuencia una inhibición para hablar de los sentimientos propios, un control de los impulsos, dentro de ellos la agresividad y el levantamiento de ideales sobre el desempeño propio, que al no ser cumplidos introducen la inconformidad. Finalmente, esta primacía del otro y del superyó trae aparejada una limitación en las capacidades para hacerle frente a las exigencias del mundo exterior. 
Respecto a la vivencia de la enfermedad por parte de los niños, se concluye que se encuentra circunscrita al interjuego formado por lo subjetivo, lo orgánico y lo social, aunque desde la perspectiva de los infantes, las mayores afectaciones estarían asociadas a los aspectos sociales. La acción de dicho interjuego se ve influenciada por las singularidades del funcionamiento psíquico de los niños entrevistados, porque si bien para ellos es posible erigir respuestas para calmar los interrogantes propios frente al proceso de enfermedad, la relación de sumisión entablada con el otro no facilita la modulación de las consecuencias sociales de la enfermedad, ni de los aspectos subjetivos que con ella se ven afectados.

El proceso de vivencia de la enfermedad en los padres da cuenta de la afectación que para ellos representa la alteración orgánica de sus hijos. En este se dan variaciones favorables que permiten la trasmudación de la incertidumbre inicial, el miedo y el temor en concepciones menos fatales de la enfermedad, en parte gracias a los cambios en la valoración del cabello y en la forma de percibir las consecuencias de la enfermedad.

Para los padres, la vivencia de la enfermedad es una tarea de varias caras, puesto que deben afrontar lo que la enfermedad de su hijo representa para sí mismo, deben contender lo que la enfermedad genera en el otro y deben ser bastión para que su hijo encare los efectos de la alopecia areata. En esta tarea muchos padres asumen ante su hijo el semblante de que la enfermedad ha sido superada, actitud que le sirve al niño, pero que da cuenta de la necesidad que tiene el padre de continuar con el afrontamiento de las renuncias narcisistas que este hecho le ha dejado. 
Num 20 - Julio-Diciembre, 2015 (Publicación preliminar) 


\section{Anexos}

\section{Producciones gráficas del Test de la Figura Humana}

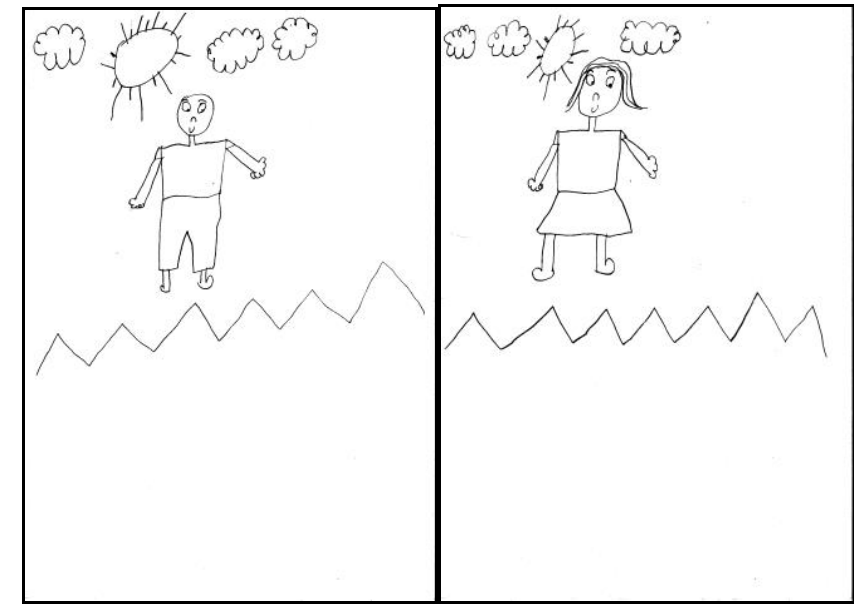

Figura 1

Figura 2

Dibujos realizados por el niño perteneciente a la familia 1, en la aplicación del Test de la Figura Humana.

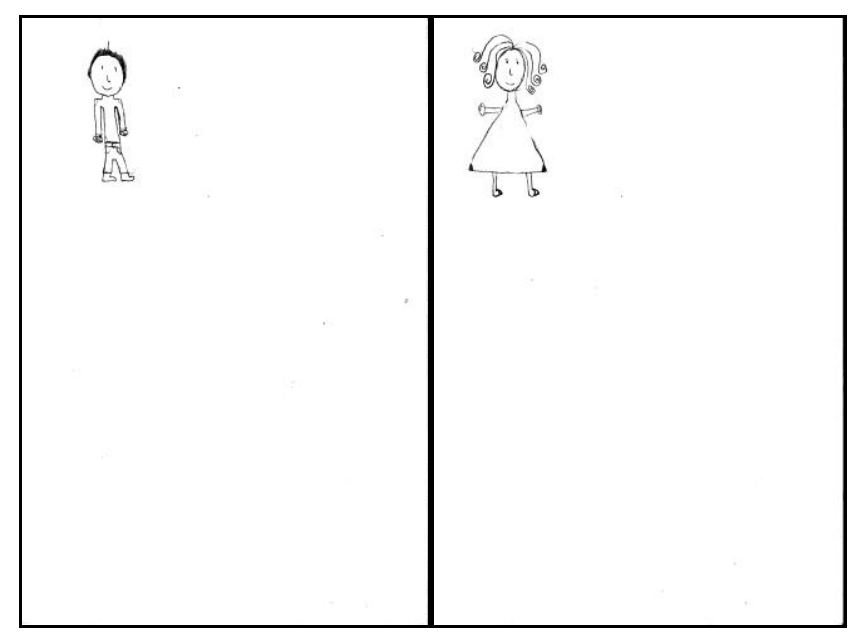

Figura 3.

Figura 4

Dibujos realizados por el niño perteneciente a la familia 2, en la aplicación del Test de la Figura Humana. 


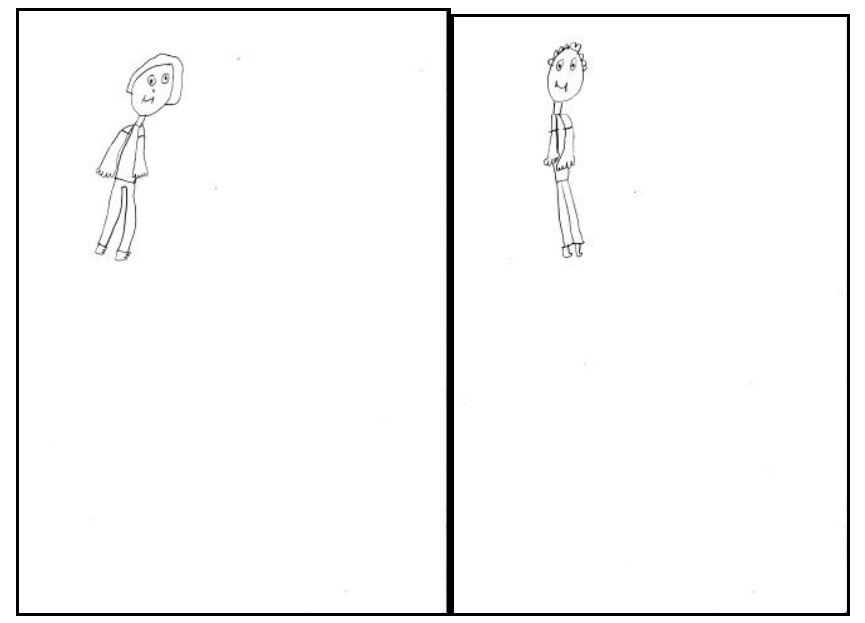

Figura 5

Figura 6

Dibujos realizados por la niña perteneciente a la familia 3, en la aplicación del Test de la Figura Humana.

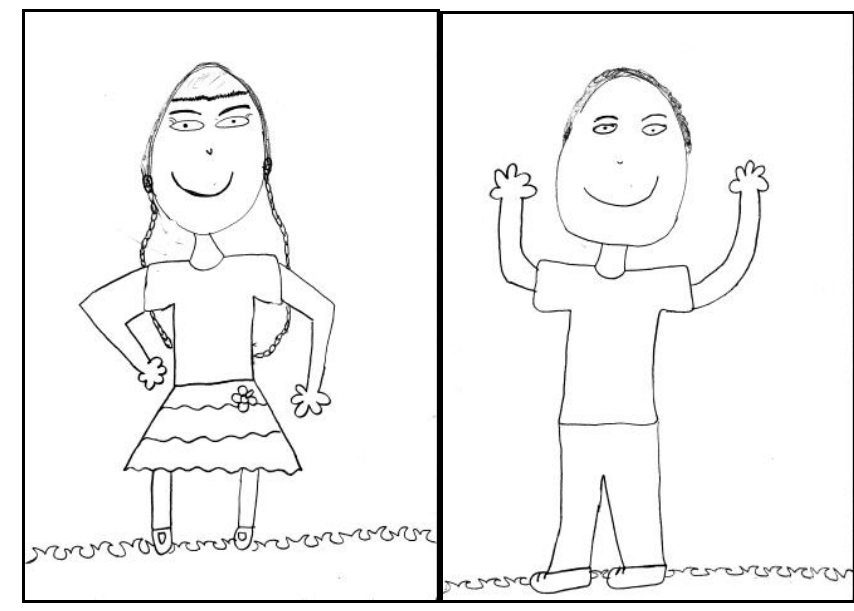

Figura 7

Figura 8

Dibujos realizados por la niña perteneciente a la familia 4, en la aplicación del Test de la Figura Humana.

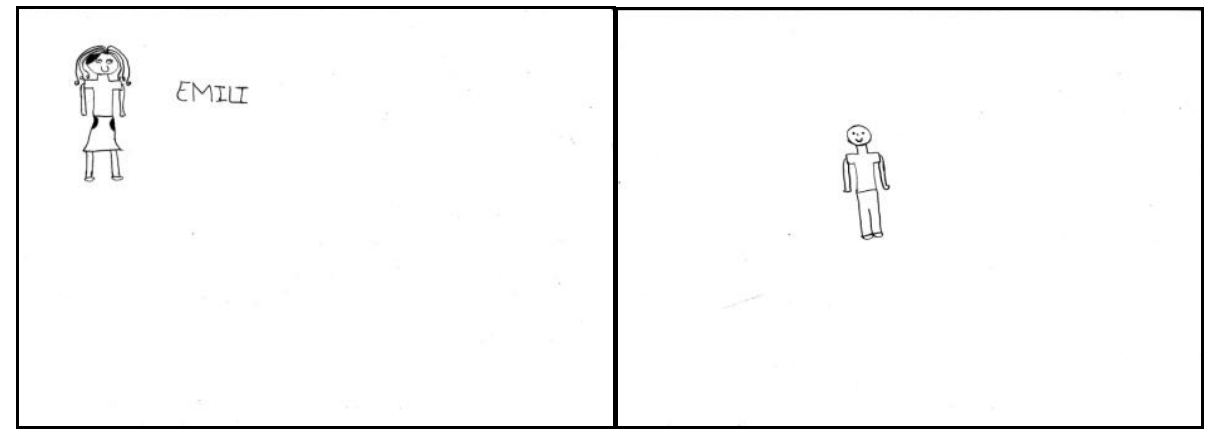


Figura 9

Figura 10

Dibujos realizado por la niña perteneciente a la familia 5, en la aplicación del Test de la Figura Humana.

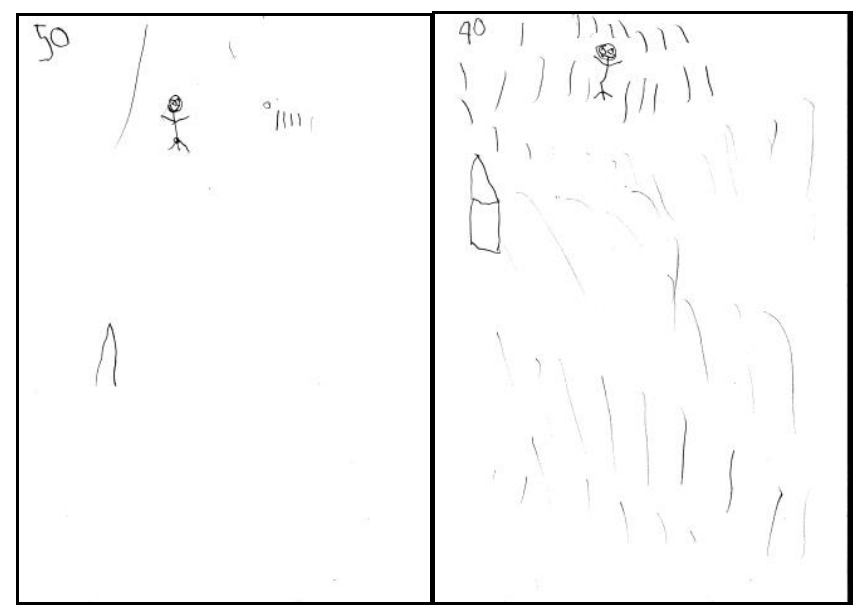

Figura 11

Figura 12

Dibujos realizados por el niño perteneciente a la familia 6, en la aplicación del Test de la Figura Humana. 


\section{Listado de referencias}

Alfani, S., Antinone, V., Mozzetta, A., Di Pietro, C., Mazzanto, C., Stella, P., Raskovich, D. \& Abeni, D. (2012) Psychological status of patients with alopecia areata. Acta derm venereal, 92, p. 304-306.

Békei, M. (1992). Coincidencias y contradicciones en las teorías psicoanalíticas sobre la psicosomatosis. Revista de Psicoanálisis, 49 (3-4), p. 543-553.

Builes Morales, A. I. \& Carmona, M. C. (1993). Estudio de casos de la relación madre-hijo en niños que presentan síntoma de vomito entre los 0 - 5 años. (Tesis inédita de pregrado). Universidad Sanbuenaventura, Medellín.

Coderch, J. (1990) Teoría y técnica de la psicoterapia psicoanalítica. Barcelona: Editorial Herder.

De Ajuriaguerra, J. (1977). Manual de psiquiatría infantil. Barcelona: Masson.

Díaz-Atienza, F. \& Gurpegui, M. (2011) Environmental stress but not subjective distress in children or adolescents with alopecia areata. Journal of psychosomatic research, 71, 102-107. doi:10.1016/j.jpsychores.2011.01.007

Farajzadeh, S., Rahnama, Z., Esfandiarpour, I., Tardast, A., Hasheminasab, S., Damavandi. F.D., Pourdamghan N. (2013). Clinical and demographic profile of childhood alopecia areata in Iran. Journal of pakistan association of dermatologists, 23 (1), p. 20-27.

Freud, S. (2004a) El yo y el ello. En J.L. Etcheverry (Traduc.), Obras completas: Sigmund Freud (Vol. 19, pp.1-63) Buenos Aires: Amorrortu (Trabajo original publicado 1923).

Freud, S. (2004b), 31 Conferencia. La descomposición de la personalidad psíquica, En J.L. Etcheverry (Traduc.), Obras completas: Sigmund Freud (Vol. 22, pp.53-74) Buenos Aires: Amorrortu (Trabajo original publicado 1933)

Galeano, M.E. (2007). Estrategias de investigación social cualitativa: el giro en la mirada. Medellín: La carreta editores. 
Ghanizadeh, A. (2008). Comorbidity of psychiatric disorders in children and adolescents with alopecia areata in a child and adolescent psychiatry clinical simple. International journal of dermatology, 47, p. 1118-1120.

Güleç, T., Tanriverdi, N., Dürü, Ç., Saray, Y. \& Akgali, C. (2004). The role of psychological factors in alopecia areata and the impact of the disease on the quality of life. International journal of dermatology, 43, p. 352-356

Hunt, N. \& Mchale, S. (2005). Reported experiences of persons with alopecia areata. Journal of loss and trauma, 10, p. 33-50. doi: 10.1080/15325020490890633.

Jurado, J. \& Ramírez, L. M. (1997). Interacción familiar y esquemas maladaptativos tempranos presentes en niños de siete a diez años de edad del hospital San Juan de Dios de la ceja, con manifestaciones psicosomáticas. (Tesis inédita de pregrado). Universidad Sanbuenaventura, Medellín.

Kakourou, T., Karachristou K. \& Chrousos G. (2007). A case series of alopecia areata in children: impact of personal and family history of stress and autoimmunity. European academy of dermatology and venereology, 21, p. 356-359. doi 10.1111/j.14683083.2006.01931.x

Kernberg, O. (1999). Trastornos graves de la personalidad. México: Manual Moderno. Kreisler, L. (1985). La desorganización psicosomática en el niño. Barcelona: Editorial Herder.

Kreisler, L., Fain, M., \& Soulé, M. (1977). El niño y su cuerpo: Estudio sobre la clínica psicosomática de la infancia. Buenos Aires: Amorrortu.

Laín Entralgo, P. (1960). La enfermedad como experiencia. En Tribuna de la Revista de Occidente 1, Experiencia de la Vida, 47-99. Madrid: Ediciones Castilla.

Leiberman de Bleichmar C., \& Bleichmar N. (2001). Las perspectivas del psicoanálisis. México: Paidos. 
Ley 1090 (2006). Título VII: Del código deontológico y bioético para el ejercicio de la profesión de psicología. Bogotá: Ministerio de la Protección Social.

Machover, K. (1974) Proyección de la personalidad en el dibujo de la figura humana. Bogotá: Ediciones Cultural.

Martínez, M. (1996) Comportamiento humano: nuevos métodos de investigación. México: Editorial Trillas.

Mercado Martínez, F. J. (1996). Entre el infierno y la gloria la experiencia de la enfermedad crónica en un barrio urbano. Guadalajara: Universidad de Guadalajara.

Koppitz, E. (1976) El dibujo de la figura humana en los niños. Evaluación psicológica. Buenos Aires: Editorial Guadalupe.

Olguín García, M.G., Del Campo, A.M., Rodríguez Acar, M., \& Peralta Pedrero, M.L. (2013). Factores psicológicos asociados con la alopecia areata. Dermatol, 57(3), 171177.

Palacios, E. (2013). Pensando desde la clínica. ¿Cómo funciona el psiquismo de los padres e hijos con predisposición a padecer manifestaciones somáticas patológicas o con patología psicosomática ya instalada? Cuatro ejemplos clínicos. Curso virtual de psicosomática infantojuvenil: un reto para la clínica psicoanálitica. Asociación psicoanalítica de Buenos Aires, Argentina.

Pasteur, R., Cuynet, P. \& Mariage, A. (2010). Psychosomatique de l'eczéma chez l'enfant: une problématique d'indifférenciation au sein du groupe familial. L'évolution psychiatrique, 75, p. 261-273. doi: 10.1016/j.evopsy.2010.04.004.

Pedreira, J.L., Palanca, I, Sardinero E., \& Martin, L. (2001). Los trastornos psicosomáticos en la infancia y la adolescencia. Revista de psiquiatría y de psicología del niño y del adolescente, 3(1), p. 26-51. 
Pérez Morais, S.T. (1999). Alopecia areata em crianças: aspectos psicológicos. Pediatria moderna, 35(3), p. 122-127.

Portuondo, J. A. (2012). La figura humana test proyectivo de Karen Machover. México: Siglo XXI.

Resolución 008430 (1993). República de Colombia: Ministerio de Salud.

Sánchez Sánchez, T. (2009). ¿Qué es psicosomática?: Del silencio de las emociones a la enfermedad. Madrid: Biblioteca Nueva.

Schmitt, J., Chen, C.M., Apfelbacher, C., Romanos, M., Lehmann, I., Herbarth, O., Schaaf, B., Kraemer, U., von Berg, A., Wichmann, H.E., Heinrich, J., \& LISA-plus Study Group. (2011). Infant eczema, infant sleeping problems, and mental health at 10 years of age: the prospective birth cohort study LISA plus. Allergy, 66, p. 404-411.

Sierra Jiménez, S. \& Torres Salazar, M. (1987). Estudio psicosomático del niño asmático. (Tesis inédita de pregrado). Universidad Sanbuenaventura, Medellín.

Strauss, A., \& Corbin, J. (2002). Bases de la investigación cualitativa: técnicas y procedimientos para desarrollar la teoría fundamentada. Medellín: Editorial Universidad de Antioquia.

Thomé Ferreira, V., Campio Müller \& Zogbi Jorge, H. (2006). Dinâmicas das relações em famílias com um membro portador de dermatite atópica: um estudo qualitativo. Psicologia em Estudo, 11(3), 617-625.

Winnicott, D. (1964) Las raíces de la agresión. En Donald Winnicott obras completas [versión Adobe Acrobat Pro]. Recuperado de http://ouricult.files.wordpress.com/2012/06/donald-winnicott-obras-completas.pdf

Winnicott, D. (1970). Vivir creativamente. En Donald Winnicott obras completas [versión Adobe Acrobat Pro]. Recuperado de http://ouricult.files.wordpress.com/2012/06/donal-winnicott-obras-completas.pdf 
Num 20 - Julio-Diciembre, 2015 (Publicación preliminar)

Winnicott, D. (1980). La familia y el desarrollo del individuo. Buenos Aires: Hormé.

Worden, W. (1997) El tratamiento del duelo: asesoramiento psicológico y terapia. Barcelona: Paidós. 